\title{
Inalação contínua com fenoterol na criança com asma aguda grave: efeitos clínicos imediatos
}

\author{
Continuous fenoterol inhalation by children with severe acute asthma: \\ immediate clinical effects
}

\author{
Lourdes Z. Zanoni ${ }^{1}$, Durval B. Palhares ${ }^{2}$
}

\section{Resumo}

Objetivos: avaliar as alterações da freqüência cardíaca, da pressão arterial, do psiquismo e da saturação arterial de oxigênio, após a inalação contínua com fenoterol, na criança com asma aguda grave.

Casuística e métodos: foram estudados 30 pacientes com asma aguda grave, atendidos no PAM-Pediatria do Hospital Universitário - UFMS. Os pacientes receberam inalação contínua durante uma hora, com $0,5 \mathrm{mg} / \mathrm{kg}$ ( 2 gotas $/ \mathrm{kg})$ de fenoterol. O psiquismo, a saturação arterial de oxigênio, a freqüência cardíaca e a pressão arterial foram avaliados antes, imediatamente após, e uma hora após a inalação com fenoterol.

Resultados: 17 crianças eram do sexo masculino $(56,6 \%)$, e 13 do sexo feminino (43,4\%). Foi observado sonolência em 16 (53,3\%), agitação psicomotora em 1 (3,3\%), náusea e vômito em 12 pacientes (40\%). A média da saturação arterial de oxigênio aumentou de 90,9 $\pm 2,8 \%$ para $92,7 \pm 2,5 \%(p<0,05)$ após a inalação. Houve um aumento estatisticamente significativo da média da freqüência cardíaca do início da inalação ao término da mesma $(139,5 \pm 13,5 \mathrm{bpm}$, $166,5 \pm 11,1 \mathrm{bpm}$, respectivamente) $\mathrm{p}<0,05$. A média da pressão arterial era de 117,56 $\pm 10,3 / 74,6 \pm 7 \mathrm{mmHg}$ antes da inalação, e ocorreu diminuição ao final da inalação, atingindo valores médios de $107,6 \pm 11 / 63,6 \pm 9,3 \mathrm{mmHg}(\mathrm{p}<0,05)$.

Conclusões: a inalação contínua com fenoterol na dose de 0,5 $\mathrm{mg} / \mathrm{kg}$, na criança com asma aguda grave, desencadeou sonolência, náusea, vômitos, taquicardia e diminuição da pressão arterial. Os autores sugerem que esta modalidade de tratamento seja realizada com monitorização clínica, em ambiente hospitalar, merecendo atenção especial aquelas crianças com doenças concomitantes, nas quais os efeitos sistêmicos da terapia poderiam ser potencializados pelas doenças subjacentes.

J Pediatr (Rio J) 2002; 78 (5): 423-8: efeitos clínicos, fenoterol, inalação contínua, asma.

1. Mestre em Medicina. Professor Adjunto do Departamento de Pediatria Universidade Federal de Mato Grosso do Sul.

2. Professor Adjunto Doutor do Departamento de Pediatria - Universidade Federal de Mato Grosso do Sul.

Artigo submetido em 23.11.01, aceito em 05.06.02.

\begin{abstract}
Objective: to evaluate the alterations of heart rate, blood pressure, psychological aspects and oxygen saturation after continuous fenoterol inhalation $(0.5 \mathrm{mg} / \mathrm{Kg})$ by children with severe acute asthma.
\end{abstract}

Methods: we studied 30 patients with severe acute asthma who were treated at the pediatric ward of Hospital Universitário - UFMS. The patients inhaled $0.5 \mathrm{mg} / \mathrm{Kg}$ of fenoterol (two drops $/ \mathrm{Kg}$ ) during one hour. Psychological aspects, oxygen arterial saturation, heart rate and blood pressure were evaluated at three different moments: before, after and one hour after the fenoterol inhalation.

Results: there were 17 males $(56.6 \%)$ and 13 females (43.4\%). Sleepiness was observed in $16(53.3 \%)$, psychomotor agitation in one $(33 \%)$ and nausea and vomiting in 12 patients $(40 \%)$. The average of oxygen arterial saturation increased from $90.9 \pm 2.8 \%$ to $92.7 \pm 2.5 \%(P<0.05)$ after inhalation. There was statistically significant increase in the average heart rate before and after inhalation (139.5 \pm 13.5 beats $/ \mathrm{min}, 166.5 \pm 11.1$ beats $/ \mathrm{min}$, respectively), $\mathrm{P}<0.05$. A significant decrease in blood pressure rate was observed from 117.56 ( $10.3 / 74.6$ ( $7 \mathrm{mmHg}$, to $107.6 \pm 11 / 63.6 \pm 9.3 \mathrm{mmHg}$ $(P<0.05)$.

Conclusions: continuous fenoterol $(0.5 \mathrm{mg} / \mathrm{Kg})$ inhalation by children with severe acute asthma caused sleepiness, nausea, vomits, palpitation and decrease in blood pressure rate. The authors suggest that patients submitted to this treatment need clinical monitorship at hospital settings. Children with concomitant diseases such as diarrhea, vomits, and dehydration require special attention.

J Pediatr (Rio J) 2002; 78 (5): 423-8: clinical effects, fenoterol, continuous inhalation, asthma.

\section{Introdução}

A asma aguda grave é caracterizada por um aumento da obstrução das vias aéreas, aumento do esforço respiratório e uma desproporção ventilação-perfusão, podendo levar à 
hipóxia, fadiga dos músculos respiratórios, retenção de dióxido de carbono e à acidose respiratória ${ }^{1,2}$.

A administração de broncodilatadores sob a forma de aerossol tem sido reconhecida como a principal forma de tratamento das exacerbações agudas da asma, devido à sua ótima biodisponibilidade, pela deposição direta da substância no seu sítio de ação na via aérea, com menos efeitos colaterais sistêmicos ${ }^{3,4}$. Estudos mostraram que a inalação de doses crescentes de albuterol produziram uma resposta broncodilatadora significativamente maior ${ }^{5}$, e que, quando administrados de forma contínua, preveniram a recorrência do broncoespasmo, permitindo uma melhor distribuição da substância nas vias aéreas periféricas, com resposta clínica mais satisfatória ${ }^{6}$. Conforme a publicação do II Consenso para o Diagnóstico e Manejo da Asma (1997) ${ }^{2}$, os broncodilatadores podem ser administrados para a criança com asma aguda sob forma de inalação contínua, na dose de 0,5 $\mathrm{mg} / \mathrm{kg} /$ hora.

Em nosso meio, o fenoterol é bastante difundido, de uso freqüente, porém estudos ainda são necessários com este medicamento quanto aos seus efeitos colaterais.

O presente estudo teve como objetivo avaliar as alterações da freqüência cardíaca, da pressão arterial, do psiquismo e da saturação arterial de oxigênio, após a inalação contínua com fenoterol, na dose de $0,5 \mathrm{mg} / \mathrm{kg} / \mathrm{hora}$, na criança com asma aguda moderada ou grave.

\section{Casuística e métodos}

Foram estudadas 30 crianças com asma aguda moderada ou grave, atendidas no Pronto Atendimento Pediátrico do Hospital Universitário da Universidade Federal de Mato Grosso do Sul. O período de estudo de cada criança foi de duas horas. O protocolo para o estudo foi aprovado pela comissão de ética médica da UFMS. A permissão de um dos pais foi obtida, e o termo de consentimento foi assinado, para a participação da criança no estudo. Foram prestadas todas as informações quanto à terapêutica a ser utilizada, inclusive a possibilidade de efeitos colaterais. As crianças com uso prévio de qualquer medicamento, portadoras de doenças cardíacas, musculares, renais ou neoplásicas não foram incluídas no estudo.

O diagnóstico clínico de asma e a intensidade da crise (Tabela 1) foram feitos de acordo com critérios estabelecidos pelo Guidelines for the Diagnosis and Management of Asthma (1991) ${ }^{1}$ e pelo II Consenso Brasileiro de Asma $(1998)^{2}$, conforme questionário descrito a seguir.

1 - Qual a idade de início, a freqüência e a duração das crises?

2 - O paciente já teve chiado no peito, ou tosse?

3 - Há piora durante o dia ou durante a noite?

4 - O paciente tem rinite, urticária, alergia a drogas ou a substâncias?

5 - O paciente melhora com broncodilatadores nebulizados ou inalados?

6 - O paciente se expõe à poeira, animais ou produtos químicos?

7 - O paciente tem crise quando corre, fica emocionado, ou durante o riso?

8 - Tem casos de alergia na família, ou fumantes em casa?

A obtenção dos dados e a avaliação clínica do paciente foram feitas por um único examinador, de acordo com a Tabela 1.

Tabela 1 - Estimativa da gravidade da crise aguda de asma na criança ${ }^{1,2}$

\begin{tabular}{|c|c|c|c|}
\hline Sinais / Sintomas & Leve & Moderada & Grave \\
\hline Freqüência respiratória & $\begin{array}{l}\text { Normal ou } 30 \% \\
\text { acima da média }\end{array}$ & $\begin{array}{l}30 \% \text { a } 50 \% \\
\text { acima da média }\end{array}$ & $\begin{array}{l}\text { Maior que } 50 \% \\
\text { acima da média }\end{array}$ \\
\hline Psiquismo & Normal & Normal & Deprimido \\
\hline Dispnéia & Ausente ou leve & $\begin{array}{l}\text { Moderada, fala frases } \\
\text { incompletas }\end{array}$ & $\begin{array}{l}\text { Intensa, fala monossílabos, } \\
\text { dificuldade } \mathrm{p} / \text { alimentar }\end{array}$ \\
\hline Musculatura acessória & Leve retração intercostal & $\begin{array}{l}\text { Moderada retração } \\
\text { intercostal, tráqueo-esternal, } \\
\text { hiperinsuflação do tórax }\end{array}$ & $\begin{array}{l}\text { Retração intercostal } \\
\text { intensa, tráqueo-esternal, } \\
\text { batimento de asas nasais }\end{array}$ \\
\hline Cor & Normal & Pálida & Cianose \\
\hline Ausculta & Sibilos expiratórios & $\begin{array}{l}\text { Sibilos inspiratórios } \\
\text { e expiratórios }\end{array}$ & $\begin{array}{l}\text { Sons respiratórios } \\
\text { inaudíveis }\end{array}$ \\
\hline Saturação de $\mathrm{O}_{2}$ & Acima de $95 \%$ & Entre $90 \%$ e $95 \%$ & Abaixo de $90 \%$ \\
\hline $\mathrm{PaCO}_{2}$ & Abaixo de $35 \mathrm{mmHg}$ & Abaixo de $40 \mathrm{mmHg}$ & Acima de $40 \mathrm{mmHg}$ \\
\hline
\end{tabular}

Os dados pulso paradoxal e pico de fluxo expiratório fazem parte desta tabela, mas não foram considerados, pois o pulso paradoxal é de difícil avaliação frente à taquicardia, e o pico de fluxo expiratório só é possível para crianças acima de 7 anos, pois essa medida depende da colaboração do paciente. Uma vez que essas medidas não poderiam ser feitas para todos os pacientes do estudo, as mesmas não foram consideradas. 
Durante o estudo, o psiquismo de cada paciente foi avaliado quanto ao surgimento de sonolência ou irritabilidade, a cada 15 minutos. Foi considerado como sonolento o paciente que dormia logo após o início da inalação, acordando apenas ao manuseio, e agitado o paciente com irritabilidade, que não aceitava a inalação, que apresentava choro sem causa aparente, como dor, medo ou afastamento da mãe.

A freqüência cardíaca foi obtida em três momentos: na chegada do paciente, ao final da inalação e depois de uma hora do término da mesma, pela leitura direta em um monitor cardíaco (Ecafix).

As pressões arteriais sistólica e diastólica foram medidas em três momentos: antes do início do tratamento, ao final da inalação e uma hora após o seu término, pelo método osciloscópio, com um aparelho da marca Dixtal, com manguito ocupando $2 / 3$ do comprimento do braço e com a criança semi-sentada. As medidas normais para cada faixa etária foram aquelas consideradas pela Second Task Force on Blood Pressure Control in Children, 198715.

A oximetria de pulso foi realizada por um oxímetro digital da marca Ohmeda, de modo contínuo, desde a chegada do paciente até o final da segunda hora.

$\mathrm{O}$ fenoterol foi administrado por meio de um nebulizador, com máscara facial e reservatório com capacidade de $20 \mathrm{ml}$, e fluxo de seis litros por minuto de ar comprimido ${ }^{16}$. A solução de nebulização foi composta de solução fisiológica $0,9 \%$ e $0,5 \mathrm{mg} / \mathrm{kg}$ de fenoterol, máximo de $15 \mathrm{mg} \mathrm{(2}$ gotas $/ \mathrm{kg}$ ), liberada para dentro do reservatório por meio de uma bomba de infusão de seringa, com fluxo de $30 \mathrm{ml}$ por hora, ajustada de modo a manter um volume de $2 \mathrm{ml} \mathrm{da}$ solução no nebulizador (adaptado de Craig et al., 1996) ${ }^{11}$, durante uma hora.

Em caso de deterioração do quadro clínico, isto é, piora da hipoxemia, com cianose, agitação psicomotora e diminuição da saturação arterial de oxigênio, em relação à entrada, a oferta de oxigênio inalatório foi imediatamente iniciada.

A análise estatística dos resultados foi feita pela média, desvio-padrão e porcentagem. O teste dos sinais dos postos de Wilcoxon ${ }^{17}$ foi usado para comparar as diferenças entre os grupos, antes e depois do tratamento. O valor $p$ adotado foi aquele menor que 0,05 . Os testes estatísticos foram realizados pelo programa Stataquest 4.0 para Windows 95 , n. ${ }^{\circ}$ de série: W-902103040, Stata Corporation, TX-USA.

\section{Resultados}

Trinta crianças foram incluídas no estudo, no período de 1998 a 2000. As características demográficas estão expressas na Tabela 2.

A sonolência foi observada mais freqüentemente aos 45 minutos do início da inalação, em 16 crianças $(53,3 \%)$. Apenas uma criança (3,3\%) apresentou agitação psicomotora aos 30 minutos do início da inalação. Náusea e vômito ocorreram em $40 \%$ dos pacientes (Tabela 3 ).
Tabela 2 - Características demográficas dos pacientes

\begin{tabular}{ll}
\hline Idade & $5,2 \pm 2,6$ anos $(2$ a 11 anos $)$ \\
Sexo & Masculino $-17(56,6 \%)$ \\
& Feminino $-13(43,4 \%)$ \\
Peso & $20,6 \pm 8,5 \mathrm{~kg}(9,5 \mathrm{~kg}-40 \mathrm{~kg})$ \\
Gravidade do quadro de asma & Moderada $-20(66,6 \%)$ \\
& Grave $-10(33,3 \%)$ \\
\hline
\end{tabular}

A média da saturação arterial de oxigênio antes do início da inalação foi de $90,9 \pm 2,8 \%$, e ao final, de $92,7 \pm 2,5 \%$ $(\mathrm{p}<0,05)$. Cinco pacientes apresentaram diminuição na saturação arterial de oxigênio após o início da inalação. Não houve correlação estatisticamente significativa entre a saturação arterial de oxigênio e a freqüência cardíaca antes e após a inalação ( $p=0,17$ e 0,38 , respectivamente).

Tabela 3 - Ocorrência de náusea e vômito durante e após a inalação com fenoterol em crianças com asma aguda grave

\begin{tabular}{ccc}
\hline $\begin{array}{c}\text { Período } \\
\text { (minutos) }\end{array}$ & $\begin{array}{c}\text { Durante } \\
\left(\mathbf{n}^{\mathbf{0}} \text { de pacientes) }\right.\end{array}$ & $\begin{array}{c}\text { Após } \\
\left(\mathbf{n}^{\mathbf{0}} \text { de pacientes }\right)\end{array}$ \\
\hline 15 & 0 & 3 \\
30 & 2 & 3 \\
45 & 1 & 1 \\
60 & 2 & 0 \\
\hline
\end{tabular}

A freqüência cardíaca aumentou progressivamente a partir do início do tratamento, atingindo o valor máximo aos 60 minutos $(p<0,05)$, coincidindo com o término da inalação com fenoterol (Tabela 4). A média do aumento da freqüência cardíaca foi de $27 \pm 12,5 \mathrm{bpm}(\mathrm{p}<0,05)$. Após o término da inalação, a freqüência cardíaca diminuiu, porém não atingiu os valores iniciais prévios ao tratamento, permanecendo $7,8 \pm 1,9$ bpm acima da média $(\mathrm{p}<0,05)$.

Tabela 4 - Valores máximo, mínimo e média da freqüência cardíaca (FC) antes, ao final da inalação com fenoterol e uma hora após o seu término, em crianças com asma aguda grave

\begin{tabular}{lccc}
\hline Período & FC (bpm) & Média \pm DP $(\mathbf{b p m})$ & Valor p \\
\hline Início & 102 a 160 & $139,5 \pm 13,5$ & $<0,05$ \\
Término & 145 a 190 & $166,5 \pm 11,1$ & $<0,05$ \\
$\begin{array}{l}\text { Uma hora após } \\
\text { o término }\end{array}$ & 105 a 163 & $147,3 \pm 11,6$ & $<0,05$ \\
\hline
\end{tabular}

Bpm: batimentos por minuto, DP: desvio-padrão 
Tanto as pressões arteriais sistólica quanto a diastólica diminuíram no final da inalação com fenoterol, com elevação progressiva após o seu término, sem, no entanto, atingir os níveis iniciais prévios ao tratamento (Tabela 5).

\section{Discussão}

Os agentes $\beta_{2}$-adrenérgicos, medicamentos de escolha para o tratamento do estado asmático, quando administrados pela via inalatória, têm alto índice terapêutico, se comparados com a administração pelas vias oral, endovenosa ou subcutânea, principalmente pelo seu efeito pulmonar local, e conseqüente minimização dos seus efeitos sistêmicos ${ }^{4,18}$. Altas doses de $\beta_{2}$-adrenérgicos passaram a ser utilizadas na criança com asma aguda grave ${ }^{8,9}$, uma vez que, frente ao reduzido calibre das vias aéreas, apenas em torno de $10 \%$ da dose desses medicamentos inalados alcançavam os pulmões ${ }^{5}$. Por promover uma estimulação contínua dos receptores $\beta_{2}$ pulmonares, a nebulização contínua pode prevenir a recorrência do broncoespasmo que ocorre com o uso intermitente, permitindo uma melhor distribuição da droga nas vias aéreas periféricas ${ }^{6}$.

A partir da observação de que doses freqüentes e maiores eram mais eficazes, a nebulização contínua passou então a ocupar um lugar de destaque no tratamento da asma aguda grave da criança $a^{9}, 10,18,19$.

Dentre os efeitos sistêmicos provocados pelos betaadrenérgicos, a taquicardia é decorrente da ação destas substâncias sobre os receptores $\beta_{2}$-adrenérgicos, existentes em pequena proporção no miocárdio ${ }^{20}$, e pela diminuição da resistência vascular periférica ${ }^{4,21}$. No grupo de crianças estudado por Schuh et al. $(1990)^{8}$, a freqüência cardíaca variou de 108 a $180 \mathrm{bpm}$, tendo ocorrido uma variação média de $33,7 \%$ com relação à freqüência da chegada. $\mathrm{O}$ valor mais alto da freqüência cardíaca, observado por Katz et al. $(1993)^{9}$, com dose de $0,5 \mathrm{mg} / \mathrm{kg}$ a cada 20 ou 30 minutos, foi de $174 \mathrm{bpm}$.

Neste estudo, a média da freqüência cardíaca apresentou uma variação significativa, com aumento progressivo durante a inalação e com diminuição gradativa até o final da segunda hora de observação (Tabela 4), sendo que esse ritmo não foi considerado como uma complicação do tratamento, e sim uma indicação do efeito farmacológico da substância sobre os receptores beta do miocárdio ${ }^{20,21}$. Uma hora após o término da inalação, a freqüência cardíaca manteve uma média acima dos valores prévios ao tratamento $(p<0,05)$, indicando que ainda estavam presentes os efeitos do fenoterol.

$\mathrm{O}$ aumento da média da saturação arterial de oxigênio no final da inalação foi estatisticamente significante, porém cinco pacientes apresentaram diminuição da saturação arterial após o início do tratamento, tendo sido iniciada oferta de oxigênio inalatório. Esta diminuição foi possivelmente decorrente da alteração da relação ventilação/perfusão, na qual o estímulo sobre os receptores $b_{2}$-adrenérgicos, induzido pelo fenoterol, leva à vasodilatação, com aumento do fluxo sangüíneo pulmonar, bem como aumento do débito cardíaco. Como relatado por Tal et al. (1984) ${ }^{22}$, um súbito aumento da perfusão sangüínea em áreas pulmonares pobremente ventiladas resultou na queda inicial da saturação arterial de oxigênio, como observado em nossos pacientes. Não houve correlação entre a saturação arterial de oxigênio e a freqüência cardíaca ao final da inalação.

Um dos efeitos adversos relacionados ao uso de fenoterol é o nervosismo ou agitação ${ }^{23}$. Neste estudo, a agitação foi observada em dois pacientes $(6,6 \%)$ e a sonolência foi um sinal mais freqüente, observada em 16 casos $(53,3 \%)$, e ocorreu predominantemente nos primeiros 45 minutos do início da inalação.

Das 30 crianças estudadas, seis apresentaram, antes da inalação com fenoterol, pressão arterial sistólica acima dos limites normais, que variou de $126 \mathrm{a} 140 \mathrm{mmHg}$, possivelmente devido à taquicardia e ao esforço físico impostos pela crise de asma.

Os resultados da pressão arterial são divergentes na literatura, como mostraram Chapman et al. $(1984)^{13}$ e Bauer et al. (1993) ${ }^{14}$, que observaram um aumento da pressão arterial sistólica e uma diminuição da pressão arterial diastólica. Diferentemente, Papo et al. $(1993)^{10}$ relataram que não houve alteração da pressão arterial no grupo de crianças por eles estudadas.

Tabela 5 - Média, valores máximo e mínimo da pressão arterial antes da inalação com fenoterol, no final e uma hora após o término da mesma em crianças com asma aguda grave

\begin{tabular}{lllll}
\hline Período & $\begin{array}{l}\text { Tempo } \\
\text { (minutos) }\end{array}$ & $\begin{array}{l}\text { Pressão arterial } \\
\text { (mmHg) }\end{array}$ & $\begin{array}{l}\text { Média e DP } \\
\text { da P.A. }\end{array}$ & Valor p \\
\hline Antes & 0 & $90 / 67$ a $140 / 85$ & $117,56 \pm 10,3 / 74,6 \pm 7,0$ & \\
Após & 60 & $77 / 43$ a $128 / 74$ & $107,6 \pm 11,0 / 63,6 \pm 9,3$ & $<0,05$ \\
Uma hora após & 120 & $100 / 70$ a $130 / 87$ & $110,8 \pm 7,3 / 70,7 \pm 8,1$ & $<0,05$ \\
\hline
\end{tabular}

P.A.: pressão arterial, mmHg: milímetros de mercúrio, DP: desvio-padrão. 
No presente estudo, a média da pressão arterial sofreu diminuição evidente e significativa no final da primeira hora, com aumento progressivo no final da segunda hora, sem, no entanto, atingir os valores iniciais. Apesar da diminuição significativa, 29 pacientes mantiveram a pressão arterial dentro dos limites considerados normais para a idade, sendo que apenas um paciente apresentou hipotensão, com níveis de $77 / 40 \mathrm{mmHg}$. Sonolência foi o único sinal clínico observado neste paciente. Quatro pacientes apresentaram diminuição da pressão arterial diastólica, que variou de 40 a $50 \mathrm{mmHg}$, níveis abaixo dos limites considerados normais para a idade; dentre esses pacientes, em três foi observado sonolência. Ao final da segunda hora de observação, a pressão arterial elevou-se novamente sem, no entanto, atingir os níveis prévios ao tratamento.

Segundo a literatura, a diminuição da pressão arterial nos pacientes em uso de beta-adrenérgicos ocorre devido a diminuição da resistência vascular periférica, induzida pela ação vasodilatadora mediada pelos beta $_{2}$-adrenérgicos ${ }^{24}$. Juntamente com a diminuição da resistência vascular periférica, é possível que a sonolência apresentada pelos pacientes tivesse colaborado para a diminuição da pressão arterial.

O surgimento de náusea e vômito, observado nos pacientes, foi interpretado como efeito adverso do fenoterol, apesar de não ter sido relatado por outros autores ${ }^{23}$.

Não foi objetivo deste estudo comparar os efeitos adversos entre as formas de inalação contínua e intermitente na criança com asma, porém nos permitiu observar que, com a inalação contínua de fenoterol, na dose de $0,5 \mathrm{mg} / \mathrm{kg}$ durante uma hora, ocorreu taquicardia sinusal, diminuição da pressão arterial, sonolência, diminuição inicial da saturação arterial de oxigênio, náusea e vômito. Os efeitos clínicos observados neste estudo não foram comparados com aqueles apresentados por outras formas de tratamento da criança com asma aguda grave, não podendo, portanto, se estabelecer comparações quanto à intensidade dos mesmos.

Os autores sugerem que a inalação contínua com fenoterol, na dose utilizada neste estudo, deve ser realizada, preferencialmente, com monitorização clínica, em ambiente hospitalar, merecendo atenção especial aquelas crianças com doenças concomitantes, como diarréia, vômitos, desidratação, nas quais os efeitos sistêmicos da terapia poderiam ser potencializados por aqueles das doenças subjacentes.

Novos estudos são necessários para verificar se os mesmos efeitos adversos observados neste estudo ocorrem com outros regimes de tratamento da criança com asma aguda grave.

\section{Referências bibliográficas}

1. Guidelines for the diagnosis and management of asthma. National Heart, Lung, and Blood Institute. National Asthma Education Program. Expert Panel Report. J Allergy Clin Immunol 1991;88(3 Pt 2):425-534.
2. Sociedade Brasileira de Pneumologia e Tisiologia, Sociedade Brasileira de Alergia e Imunologia; Sociedade Brasileira de Pediatria. II Consenso Brasileiro no Manejo da Asma, 1998.

3. Brain J, Valberg PA. Deposition of aerosol in the respiratory tract. Am Rev Respir Dis 1979;120:1325-73.

4. Clark DJ, Lipworth BJ. Dose-response of inhaled drugs in asthma. Clin Pharmacokinet 1997;32:58-74.

5. Nelson HS, Spector SL, Whitsett TL, George RB, Dwek JH. The bronchodilator response to inhalation of increasing doses of aerosolized albuterol. J Allergy Clin Immunol 1983;72:371-5.

6. Moler FW, Hurwitz ME, Custer JR. Improvement in clinical asthma score and $\mathrm{PaCO}_{2}$ in children with severe asthma treated with continuously nebulized terbutaline. J Allergy Clin Immunol 1998;81:1101-9.

7. Maguire JF, O'rourke PP, Colan SD, Geha RS, Crone R. Cardiotoxicity during treatment of severe childhood asthma. Pediatrics 1991;88:1180-6.

8. Schuh S, Reider MJ, Canny G, Pender E, Forbes T, Tan YK, et al. Nebulized albuterol in acute childhood asthma: comparison of two doses. Pediatrics 1990;86:509-13.

9. Katz IA, Irwig L, Vinen JD, March L, Wyndham LE, Luu T, et al. Biochemical markers of acute myocardial infarction: strategies for improving their clinical usefulness. Ann Clin Biochem 1998;35:393-9

10. Papo MC, Frank J, Thompson AE. A prospective randomized study of continuous versus intermittent nebulized albuterol for severe status asthmaticus in children. Crit Care Med 1993;21:1479-86.

11. Craig VL, Bigos D, Brilli R J. Efficacy and safety of continuous albuterol nebulization in children with severe status asthmaticus. Pediatr Emerg Care 1996;12:1-5.

12. Crane J, Burgess C, Beasley R. Cardiovascular and hypokalaemic effects of inhaled salbutamol, fenoterol, and isoprenaline. Thorax 1989;44:136-40.

13. Chapman KR, Smith DL, Rebuck AS, Leenen FHH. Hemodynamic effects of inhaled beta-2 agonist. Clin Pharmacol Ther 1984;35:762-75.

14. Bauer KG, Kaik B, Sertl K, Kaik GA. Studies on bronchodilator, tremorogenic, cardiovascular and hypokalaemic effects of fenoterol dry powder in asthma. Br J Clin Pharmac 1993; $36: 285-92$.

15. National Heart, Lung, And Blood Institute. Report of the second task force on blood pressure control in children - 1987. Pediatrics 1987;79:1-25.

16. Newman SP, Clarke SW. Therapeutic aerosols 1- physical and practical considerations. Thorax 1983;38:881-6.

17. Daniel WW. Biostatistics: a foundation for analysis in the health sciences. $6^{\mathrm{a}}$ ed. New York: John Wiley \& Sons. Inc.; 1995.

18. Katz RW, Kelly W, Crowley MR, Grad R, Mcwilliams BC, Murphy SJ. Safety of continuous nebulized albuterol for bronchospasm in infants and children. Pediatrics 1993;92:666-9.

19. Portnoy J, Nadel G, Amado M, Willsie-Ediger S. Continuous nebulization for status asthmaticus. Ann Allergy 1992;69:71-9.

20. Emilien G, Maloteaux JM. Current therapeutic uses and potential of b-adrenoceptor agonists and antagonists. Eur J Clin Pharmacol 1998;53:389-404.

21. Newnham DM, Wheeldon NM, Lipworth BJ, Mcdevitt DG. Single dosing comparison of the relative cardiac $\beta_{1} / \beta_{2}$ activity of inhaled fenoterol and salbutamol in normal subjects. Thorax 1993;48:656-8

22. Tal A, Pasterkamp H, Leahy F. Arterial oxygen desaturation following salbutamol inhalation in acute asthma. Chest 1984;86:868-9. 
23. Heel RC, Brogden RN, Speight TM, Aver YGS. Fenoterol: a review of its pharmacological properties and therapeutic efficacy in asthma. Drugs 1978;15:3-32.

24. Newhouse MT, Chapman KR, Mccallum AL, Abboud RT, Bowie DM, Hodder R, et al. Cardiovascular safety of high doses of inhaled fenoterol and albuterol in acute severe asthma. Chest 1996;110:595-603.
Endereço para correspondência:

Dra. Lourdes Z. Zanoni.

Rua Alexandre, 378 - Bairro Giocondo Orsi

CEP 79022-080 - Campo Grande, MS

Fone: (67) 351.8820 - E-mail: 1zzanoni@nin.ufms.br 